\title{
An Investigation of the Effects of Chemical Reagents on the Shear Bonding Forces of Orthodontic Metal Brackets
}

\author{
Chien-Chih $\mathrm{Yu}^{1}$, Jian-Hong $\mathrm{Yu}^{2,3^{*}}$ and Hsiu-Ju Lin ${ }^{3}$ \\ ${ }^{1}$ School of Pharmacy, College of Pharmacy, China Medical University, Taichung, 40402, Taiwan \\ ${ }^{2}$ School of Dentistry, College of Medicine, China Medical University, Taichung, 40402, Taiwan \\ ${ }^{3}$ Orthodontics, Department of Dentistry, China Medical University Hospital, Taichung, 40402, Taiwan \\ ${ }^{4}$ Graduate Institute of Clinical Medicine, College of Medicine, China Medical University, Taichung, 40402, Taiwan
}

"Corresponding author: Jian-Hong Yu, School of Dentistry, China Medical University 91, Xue-Shi Road, North District, Taichung City, 40402, Taiwan, Tel: +04 22053366; Fax: 042230 4333; E-mail: kenkoyu@mail.cmu.edu.tw

Rec date: June 16, 2015; Acc date: June 01, 2016; Pub date: June 10, 2016

Copyright: @ 2016 Yu CC, et al. This is an open-access article distributed under the terms of the Creative Commons Attribution License, which permits unrestricted use, distribution, and reproduction in any medium, provided the original author and source are credited.

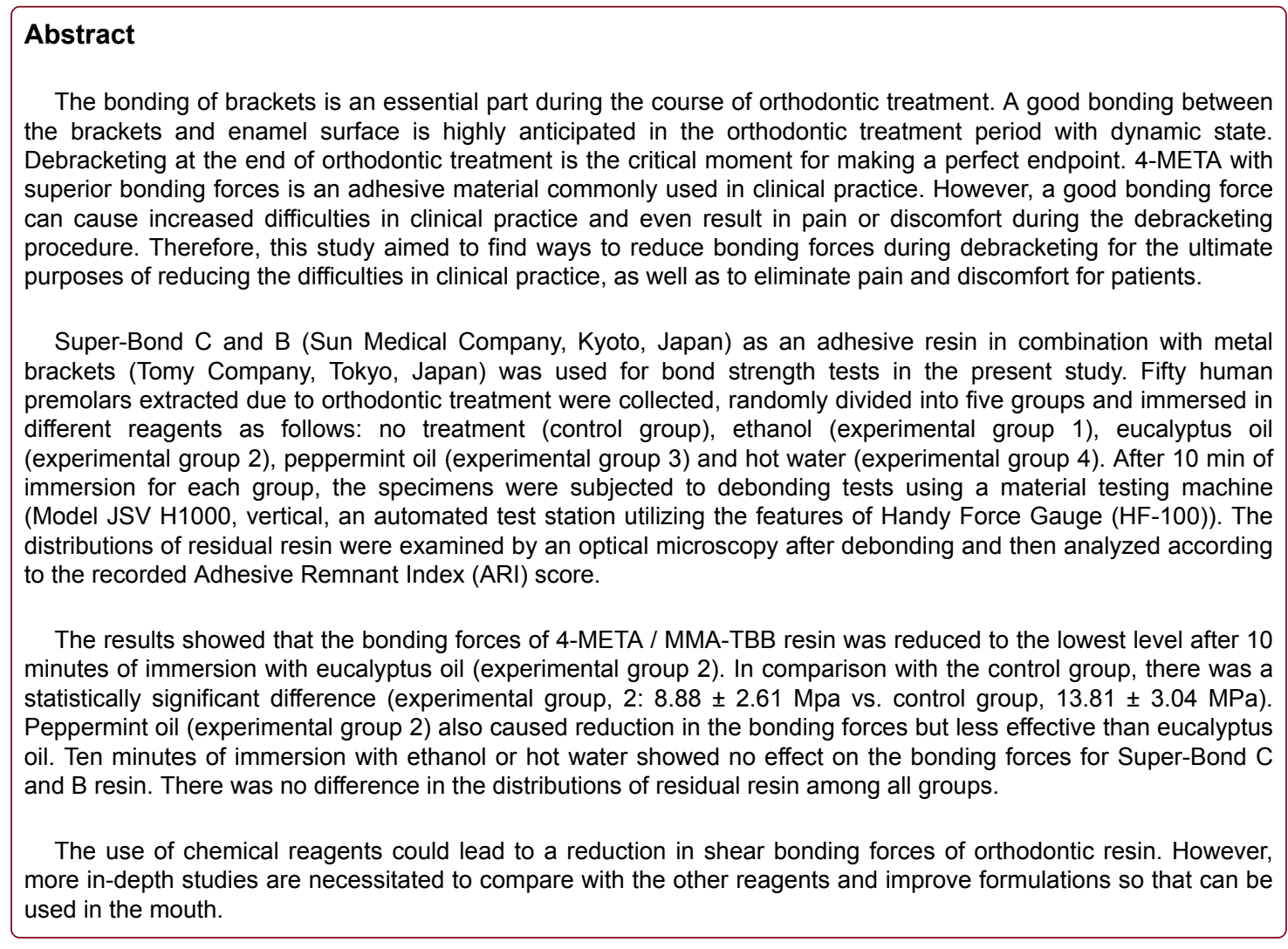

Keywords: Chemical reagents; Shear bonding forces; Metal brackets

\section{Introduction}

A complete course of orthodontic treatment usually takes one to two years. Enhancing the bonding forces is always considered important in the development of orthodontic adhesives. However, at the end of orthodontic treatment, the superior bonding forces may lead to problems during debracketing [1]. This study therefore attempted to resolve the dilemmas that evoke distress for patients and clinicians. To meet patient's expectations in clinical practice, we expected to achieve a decrease in the bonding forces of adhesive resins without the use of any additional equipment or device but by simply applying reagents to reduce patient's discomfort during the debracketing procedure $[2,3]$.

The bonding force of 4-META/MMA-TBB resin is remarkable and meets the clinical needs. PMMA-based resins can also be easily removed and left no residue on enamel surface after debonding, which has been the top priority among orthodontic adhesives. The properties of PMMA-based resins include intolerance to high temperature, nonresistant to organic solvents, soluble in organic solvents and swelling in alcohols.

Considering the special characteristics of PMMA resins, the effects of ethanol, eucalyptus oil, peppermint oil, and hot water on the orthodontic bonding forces between metal bracket and 4-META/ 
MMA-TBB resin was investigated in an attempt to find clinically effective reagents for reducing bonding forces [4].

\section{Material and Methods}

\section{Materials}

The chemical reagents used in this study include ethanol, peppermint oil and eucalyptus oil. The influence of temperature (hot water) was also considered in addition to these three chemicals.

The other materials used in the present study consisted of extracted human premolar teeth, Super-Bond C and B (4-META/MMA-TBB resin), metal brackets (Tomy Micro-arch) [5].

Extracted human premolar teeth: A total of 50 extracted human premolar teeth were collected according to the following criteria:

- The upper and lower premolar teeth extracted due to orthodontic treatment.

- The teeth with intact tooth crowns.

- No large cavity, dental filling or crack that can affect the strength of enamel.

- No tooth decay, dental filling or damage on buccal side of tooth (the surface to be bonded with bracket).

- The teeth had not been pre-treated with chemical agents.

The residual impurities or calculus on tooth surfaces were removed with ultrasonic scaler after collection, followed by storing in distilled water at room temperature to avoid dehydration and damage to the tooth surfaces.

Metal brackets: Preadjusted brackets, Micro-arch, Roth type, 0.018 slot metal brackets (Tomy Company, Tokyo, Japan). The base area of maxillary premolar bracket was $11.215 \mathrm{~mm}^{2}$ and the base area of mandibular bracket was $10.725 \mathrm{~mm}^{2}$.

Orthodontic adhesive: 4-META/MMA-TBB resin (Super-Bond C and B, Sun Medical Company, Kyoto, Japan).

\section{Methods}

A material testing machine was used to measure and record the force required during the debracketing procedure in this study [6]. Each tooth was embedded on the specimen fixture so that the tooth could be positioned on the material testing machine. The real situations of teeth fixed within the alveolar bone in human oral cavity were simulated by the use of resin as specimen fixture in embedding procedures. This procedure was also for avoiding experimental errors due to specimen movement which was caused by the force exerted by material testing machine [7-10]. After the teeth were carefully embedded, the specimens were then placed in a sink prefilled with distilled water. All bonding procedures were performed by the same operator according to the manufacturer's instructions as in Figure 1.

There were five groups in this study, including one control and 4 experimental groups. Each group was subjected to one different liquid reagent as follows:

- Control group: distilled water at room temperature

- Experimental group 1: 95\% ethanol

- Experimental group 2: Eucalyptus oil

- Experimental group 3: Peppermint oil

- Experimental group 4: Hot water at $55 \sim 60^{\circ} \mathrm{C}$

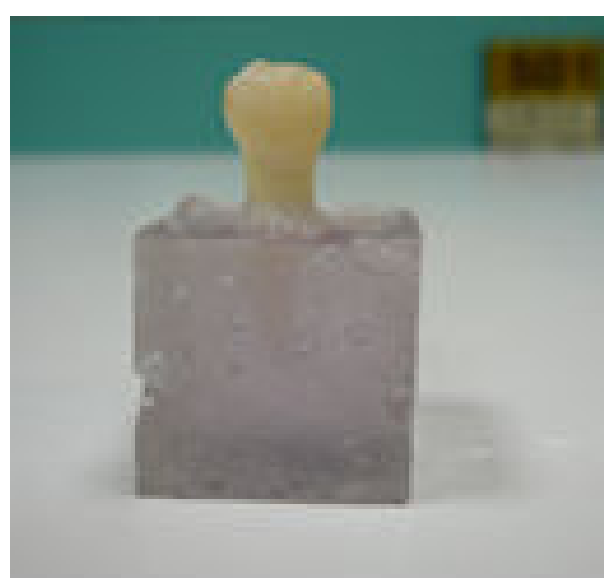

Figure 1: The representive graph of an embedded tooth

The designed experimental flow chart was as follows in Figure 2.

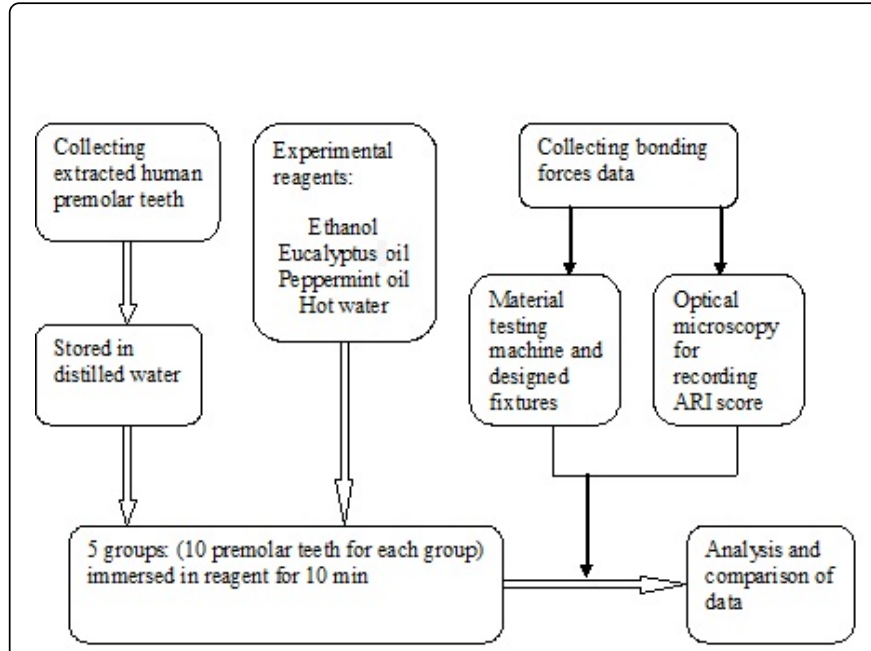

Figure 2: Experimental flow chart

A material testing machine with the use of QC Force software was used for determining and recording the changes in debonding force levels and debonding time required during the debracketing procedure. The enamel surface conditions after debracketing were observed using an optical microscopy. The distributions of residual resin were examined and analyzed with ARI score.

Material testing machine used in this study (Model JSV H1000, Vertical) was an automated test station utilizing the features of Handy Force Gauge (HF-100), with a maximum load capacity of $1000 \mathrm{~N}$ and a minimum resolution of 1/10000. Data of test results were transferred to a computer and processed with QC Force software.

The distribution patterns of residual resin on bracket bases were examined using an entity image microscope after debracketing using the material testing machine [11]. The areas with residual resin on bracket bases and teeth surface were analyzed with the aid of Petrographic Image Analysis (PIA) software. Adhesive Remnant Index (ARI) score was used to examine and classify the distribution patterns 
of residual resin [12]. ARI scoring system firstly developed by Bergland and Artun which was applied to evaluate the distributions of residual resin left. The scoring criteria of ARI were as follows (0-3): score 0: no adhesive left on the tooth; score 1: less than half of the adhesive left on the tooth; score 2: more than half of the adhesive left on the tooth and score 3 = all adhesive left on the tooth. Analysis of the data was performed using the Statistical Package for Social Science version 13.0 for windows (SPSS Inc., Chicago, IL, USA) in this study [13].

\section{Results}

Results of this study were divided into three parts, including the recorded debonding force levels required to debracket by the material testing machine, the total time needed to debracket acquisited using QCForce, and the ARI score calculated according to the residual resin left on bracket base and enamel surface determined by an optical microscope.

\section{The debonding forces measured by material testing machine}

The results showed that the maximum debonding force required to debracket for the control group and experimental groups treated with $95 \%$ ethanol, eucalyptus oil, peppermint oil and water were $152.43 \pm$ $34.23(\mathrm{~N}), 146.2 \pm 44.97(\mathrm{~N}), 97.46 \pm 29.01(\mathrm{~N}), 118.43 \pm 21.39(\mathrm{~N})$ and $146.19 \pm 36.13(\mathrm{~N})$ respectively. The minimum debonding force required was found in the group treated with eucalyptus oil for $10 \mathrm{~min}$ before debonding [14]. The teeth collected in this study consisted of and maxillary and mandibular teeth. The base areas of maxillary and mandibular brackets were $11.215 \mathrm{~mm}^{2}$ and $10.725 \mathrm{~mm}^{2}$, respectively. Almost no difference in base area between the two brackets was found. For the purpose to more precisely evaluate the changes in shear bonding forces (Mpa), the measured maximum debonding force was divided by the base area of bracket for each specimen. The bond strength values measured for each group were $13.81 \pm 3.04 \mathrm{Mpa}$ (control group), $13.24 \pm 3.94 \mathrm{MPa}$ (experimental group 1: ethanol), $8.88 \pm 2.61 \mathrm{MPa}$ (experimental group 2 : eucalyptus oil), $10.71 \pm 1.98$ $\mathrm{MPa}$ (experimental group 3: peppermint oil) and $13.25 \pm 3.17 \mathrm{MPa}$ (experimental group 4: hot water) $[15,16]$.

\section{The analysis of time needed to debracket using the material testing machine}

The results obtained after analysis were $63.427 \pm 40.36$ seconds (control group), $34.329 \pm 8.91$ seconds (experimental group 1: ethanol), $25.574 \pm 12.26$ seconds (experimental group 2: eucalyptus oil), $29.266 \pm 7.87$ seconds (experimental group 3: peppermint oil) and $41.621 \pm 18.66$ seconds (experimental group 4 : water) respectively [17].

\section{Analysis of ARI scores for the residual resin}

After finishing the test at debonding, the removed brackets were examined for the distributions of residual resin by an optical microscope. Assessment of damages on the enamel surfaces were performed according to ARI after debracketing [18]. The obtained mean ARI scores are shown in the following Table 1.

\begin{tabular}{|l|l|l|l|l|l|}
\hline & Control & Ethanol & $\begin{array}{l}\text { Eucalyptus } \\
\text { oil }\end{array}$ & peppermint oil & $\begin{array}{l}\text { Hot } \\
\text { water }\end{array}$ \\
\hline ARI score & 0.8 & 0.7 & 0.5 & 0.7 & 0.5 \\
\hline
\end{tabular}

Table 1: The ARI scores in each group after debracketing
The results showed no difference in ARI scores among all groups, with the mean values all less than 1 . In addition, most of the enamel surfaces revealed no obvious/visible damage or fracture after debracketing. Enamel fractures were only observed in three specimens, which were from control group, experimental group 1 (ethanol) and experimental group 4 (hot water).

\section{Discussion}

\section{The bracket debonding forces}

The effects of different reagents on bracket bonding forces were explored in this study. The results indicated that all the strength values obtained for experimental groups were lower than control group. However, only the group treated with eucalyptus oil (experimental group 3 ) showed significant difference $(\mathrm{p}<0.05)$ after statistical analysis $[19,20]$.

First of all, the maximum debonding force was $146.2 \pm 44.97(\mathrm{~N})$ in the group treated with ethanol (experimental group 1), which was converted to the shear bonding forces in megapascals and reported as $13.24 \pm 3.95 \mathrm{Mpa}$. The results showed almost no difference when compared to control group, indicating that ethanol is a very mild solvent and does not affect acrylic resin in a short period of time. Ehanol was also one of reagents used in the study reported by Larmour. The measured bracket debonding forces was $91.9 \mathrm{~N}$ after immersed in ethanol for one hour, which was slightly lower than control group $(103.7 \mathrm{~N})$ but without reaching statistical significance. The orthodontic adhesive resin used in our study was different from that used in Larmour's experiments [21]. However, the results obtained in our study were similar to Larmour's, showing that no significant effect was observed after immersing the specimens in ethanol within a short period of time.

Furthermore, we had attempted to immerse the debonded brackets in ethanol for $24 \mathrm{hrs}$. in a previous pilot study. The residual resin on bracket base was softened and rendered a separable state. In addition, according to a previous study investigating the effects of chemicals on orthodontic adhesive resin conducted by Yap et al., the orthodontic resin was softened after immersion in ethanol solution for a week. The results of hardness test were also significantly different when compared to control group. Therefore, ethanol solution should have potential impact on orthodontic resin materials. Long-term immersion would lead to softening and denaturation [22,23]. However, the effect of a short-term immersion (10 minutes to 1 hour) was insufficient to induce significant changes in the bonding forces of orthodontic bracket adhesive resin.

The mean debonding force of experimental group 3 (peppermint oil) was $118.43 \pm 21.39 \mathrm{~N}$ in this study. The maximum debonding force of experimental group 3 (peppermint oil) was lower than the debonding force of $152.23 \pm 34.23 \mathrm{~N}$ obtained from control group [24]. However, the difference was not statistically significant. Peppermint oil product was commercially available in 1991. At that time, manufacturers claimed that it could effectively reduce shear bonding forces in only 60 seconds. However, no significant change was found in Larmour's study for the bonding forces of specimens treated with peppermint gel product for 5 minutes. Treatment with peppermint oil product for one hour was required to achieve significant reduction in shear bonding forces. In this study, our results indicated that the overall bonding forces of Super-Bond C and B was lower than the control group after treatment with peppermint oil for 10 minutes, but 
did not yet reach a statistically significant difference. The findings might be correlated to limited samples (10). More precise data may be obtained if the sample size was increased [25].

When compared with previous studies, one hour of immersion in peppermint oil was required to lower bonding forces in previous findings. The results of Larmour's study showed that the bonding forces of control group and one-hour peppermint oil-treated group were $103.7 \pm 37.1 \mathrm{~N}$ and $7.0 \pm 18.9 \mathrm{~N}$, respectively. No significant difference was observed in ANOVA analysis. But Larmour still concluded that the maximum debonding force was effectively reduced by peppermint oil [26]. Our findings revealed the same observations, showing the maximum debonding force was lowered after the use of peppermint oil. However, the immersion duration was only 10 minutes in our study, and that was 1 hour in Larmour's. There may be two reasons led to difference in obtained results:

- The resins used were different. Photo-polymerized composite resin with filler was used in Larmour's study, whereas we used the resin made of pure PMMA (Super-Bond) containing no other material in this study. The addition of inorganic filler or particles was likely to cause differences in their properties in spite of both were methacrylate-based resins, implying it was inappropriate to compare the two resins from this standpoint.

- Reagent formulations were different. Peppermint oil was formulated with gel in previous report; however, we used the liquid form of peppermint oil in this study [26]. The specimens were immersed in peppermint oil, leading the penetration of peppermint oil into bracket-adhesive interface and consequently achieving an effect on the bonding forces in deeper layer/interface. As a result, peppermint oil used in liquid form showing an effective impact on bonding forces in a shorter period of time.

And why peppermint oil could effectively reduce the shear bonding forces? A previous study conducting a hardness test has shown that resin was not softened after immersed in peppermint oil from 0 to 180 seconds. We have neither found the softening of orthodontic adhesive resin with the naked eye or an optical microscope after immersion in peppermint oil [27]. However, the immersion duration was $10 \mathrm{~min}$ in our experiment, which was much longer in comparison with $3 \mathrm{~min}$ in the previous study. There might be different findings if specimens were subjected to hardness tests. It is recommended to conduct in depth investigations of the mechanisms underlying the effects of peppermint oil on resin in the future.

In addition, our results show that the effectiveness of experimental group 2 (eucalyptus oil) was the most significant and reached statistically different. The mean debonding force required was $102.82 \pm$ $32.76 \mathrm{~N}(8.88 \pm 2.61 \mathrm{MPa})$, which was reduced to $2 / 3$ after the use of eucalyptus oil. Chloroform was once tested in our pilot study. The obtained results indicated that the required debonding force was significantly reduced to $1 / 3$ after $10 \mathrm{~min}$ of immersion [28]. However, the use chloroform was eventually abandoned for safety considerations. Eucalyptus oil with similar effectiveness in dissolving root canal filling material was used in this study as an alternative. Eucalyptus oil preparations contain $75 \sim 80 \%$ eucalyptol (1, 8-cineole). Despite of the fact that eucalyptus oil has a very long history of use in root canal treatment and is considered the priporitized alternative reagent for chlorofrom, all the research literatures related to eucalyptus oil were focusing on comparing the dissolving effects on canal filling materials but none for its impact on resin. However, eucalyptus oil dissolving PMMA resin was firstly disclosed in this study. A significant difference in reducing bonding forces compared to other experimental groups was observed.

Finally, the impact of hot water (experimental group 4) on orthodontic bonding forces was discussed. Taking into account the tolerable temperature in mouth, hot water at $55 \sim 60^{\circ} \mathrm{C}$ was used is this study. Rueggenberg and Lockwood also reported that the force required for debonding was reduced by approximately half by raising the temperature of the bracket adhesive interface to $52^{\circ} \mathrm{C}$ [29]. The debonding force required was $146.19 \pm 36.13(\mathrm{~N})$ after immersion in hot water for $10 \mathrm{~min}$, showing almost no difference to control group in our study. Only rely on the heat of hot water to raise the temperature of bracket-adhesive interface is not easy. Although the water temperature was higher than $52^{\circ} \mathrm{C}$, it remained insufficient to elevate the temperature of bracket-adhesive interface for facilitating the melting of adhesive resin.

However, by the use of temperature to reduce the bonding forces of PMMA resin was indeed a feasible approach. In 2007, a study was conducted with the application of heat on reducing the bonding forces of Super-Bond C and B. They mixed the PMMA particles with microcapsules that released heat and the temperature was raised up to $80^{\circ} \mathrm{C}$, which consequently reduced the bonding forces of Super-Bond. It suggests that the current trend and leading practice are to timely lower the bonding forces in orthodontic materials applications. Raising the temperature by thermal heating has also been a method considered by many researchers. However, the bonding force of orthodontic adhesive resin was not reduced by simply using the heat produced by hot water within a short time in our study.

Furthermore, the thinking may be moved in the opposite direction. Orthodontic treatment is usually a long-term therapy. The duration of 1 to 2 years is required for the bonding of orthodontic brackets to teeth. The long-term dietary exposure to food variability and foods with high temperature can result in potential effects on the bracketadhesive interface.

\section{The time length required for debracketing}

In addition to pain caused by excessive force, the difficulties associated with debracketing in clinical practice should also consider the time length consumed in debracketing procedure. Patients sometimes have to endure uncomfortable feeling that is relatively extended over the debracketing process. Therefore, we also made an attempt to compare the time length required for debracketing [30,31].

The results demonstrated that the time length required to debracket were shorter in all experimental groups than $63.427 \pm 40.36$ seconds of control group. The followings were in order of time length for experimental groups: $41.621 \pm 18.66,34.329 \pm 8.91,29.266 \pm 7.87$ and $25.574 \pm 12.26$ seconds for hot water, ethanol, peppermint oil and eucalyptus oil, respectively. Comparing the findings with the orders of maximum force required for debonding, we found the effectiveness of peppermint oil and eucalyptus oil were very clear. For the application of orthodontic PMMA resin, the use of peppermint oil and eucalyptus oil not only lowered the debonding force required, but also significantly reduced the time length of debracketing [32]. Furthermore, in spite of the force for debracketing was unaffected after the specimens were immersed in ethanol, the time length required for debonding was approximately half of control group and even shorter than the group treated with hot water. Therefore, although the use of ethanol was not helpful in lowering debonding force, it showed a potential to reduce the time length required for debracketing [33]. 
Our study on the time length of debractekting procedure did not apply a constant force, but involving an overall changes in time length and force. The larger the debonding force required, indicating a relatively longer time of debracketing was needed. It may be more appropriate to interpret the force change within a time unit by using the viewpoint of slope. The results of force changes in per unit time were $3.118 \pm 1.53 \mathrm{~N}, 4.309 \pm 0.89 \mathrm{~N}, 4.327 \pm 1.13 \mathrm{~N}, 4.711 \pm 1.22 \mathrm{~N}$ and $4.00 \pm 1.46 \mathrm{~N}$ for control group and experimental groups using alcohol, eucalyptus oil, peppermint oil and hot water, respectively. There was no significant differences among all groups, showing the force change in per unit time for PMMA resin was 3-4 N/second.

If the time length was compared based on the same force applied to debracket, the resulting data should be a more meaningful index.

\section{Investigation on the distributions of residual resin}

ARI scores obtained were fairly the same for all groups in this study. All of the mean values were less than 1 . The results indicated that the debracketing procedure did not result in residual resin while bonding orthodontic brackets was used with Super-Bond C and B, which was consistent with the findings in clinical practice. In addition to high bonding forces of Super-Bond C and B in clinical use, the polymerization of Super-Bond C and B also exhibits a distinguishing feature. It is characterized by linear polymerization of monomers and contains no additional inorganic particles/fillers [34]. As a result, it was easy to be cleaned and the residue was hardly found on the tooth surface after debracketing.

Due to its superior material properties, the use of Super-Bond C and $\mathrm{B}$ in bonding forces tests for orthodontic brackets led to no change in the distributions of residual resin under the conditions with or without chemical reagents.

The results also indicated that the bonding forces between the bracket base and Super-Bond C and B was greater than that between Super-Bond C and B and the enamel. The brackets made of the base metals were used in this study. The bonding forces between SuperBond and precious metals are not strong, which needs the use in combination with other adhesive to achieve the desired bonding forces. In contrast, Super-Bond contains 4-META that can form chemical bonds with oxidized metals, leading to the superior bonding forces with base metals. Due to the metal brackets were used in this study, a reasonable result was obtained.

There were 3 specimens with visible cracks in enamel, one each from control group, experimental groups treated with ethanol and hot water. However, no significant difference in the debonding force was obtained, with all about $140 \sim 150 \mathrm{~N}$. According to the report of Bishara, it was suggested to avoid applying any debonding force greater than $13.53 \mathrm{Mpa}$ for preventing tooth enamel from unnecessary damage. The results concluded that the excessive debonding force may lead to unwanted damage to the teeth [35-37].

\section{Results of this study were concluded as follows}

- After the brackets were immersed in eucalyptus oil for 10 minutes, the bonding forces of Super-Bond C and B (4-META / MMA-TBB resin) was reduced and consequently led to the minimum force required for debonding [38]. The damage of tooth enamel resulted from debracketing procedure was also lowered.
- Peppermint oil lowered the bonding forces for brackets, but less effectively than eucalyptus oil. Ethanol and hot water did not affect the bonding forces for Super-Bond $\mathrm{C}$ and B resin.

- When Super-Bond C and B (4-META / MMA-TBB resin) was used in orthodontics for bonding brackets, leaving an almost clean enamel surface after debracketing. It suggests that Super-Bond C and $B$ is very suitable for orthodontic bracket bonding $[39,40]$.

\section{Future Prospects}

The subject regarding techniques used for reducing the bonding forces during debracketing is drawing more and more attention. Although this study attempting to investigate the methods with the use of chemical reagents for reducing bonding forces was not the first or new, most of the related information remains unknown. Only a few chemicals commonly used in orthodontics were tested as research materials in this study. There are still a very wide range of possibilities for organic solvents that we may be unable to prove their effectiveness. Apart from making improvements on reagent formulations in subsequent research studies, we will also look for different types of chemical reagents for comparison and expect to identify the most safe and effective chemical reagents.

\section{References}

1. Hotta K, Mogi M, Miura F, Nakabayashi N (1992) Effect of 4-MET on bond strength and penetration of monomers into enamel. Dent Mater 8: 173-175.

2. Chen PT, Chen CS, Gung YW (2008) Failure analysis of enamel after removing orthodontic bracket. National Yang-Ming University, Institute of Rehabilitation Science and Technology, Master Thesis.

3. Nicholson JW (2000) Adhesive dental materials and their durability. International Journal of Adhesion \& Adhesives 20: 11-16.

4. Kusy RP (2002) Orthodontic biomaterials: from the past to the present. Angle Orthod 72: 501-512.

5. Faust JB, Grego GN, Fan PL, Powers JM (1978) Penetration coefficient, tensile strength, and bonding forces of thirteen direct bonding orthodontic cements. Am J Orthod 73: 512-525.

6. Brantley WA, Eliades T (2001) Orthodontic materials: Scientific and clinical aspects. Thieme, New York pp: 190-191.

7. Bayne SC (2005) Conservative Operative Dentistry. Lecture: Bonding Systems.

8. No auhors (2005) Shin Yean Orthodontic Products.

9. Brosh T, Kaufman A, Balabanovsky A, Vardimon AD (2005) In vivo debonding forces and enamel damage in two orthodontic debonding methods. Journal of Biomechanics 38: 1107-1113.

10. Katona TR, Long RW (2006) Effect of loading mode on bonding forces of orthodontic brackets bonded with 2 systems. Am J Orthod Dentofacial Orthop 129: 60-64.

11. Norevall LI, Marcusson A, Persson M (1996) A clinical evaluation of a glass ionomer cement as an orthodontic bonding adhesive compared with an acryl ic resin. Eur J Orthod 18: 373-384.

12. Mandall NA, Millett DT, Mattick CR, Hickman J, Worthington HV, et al. (2002) Orthodontic adhesives: a systematic review. J Orthod 29: 205-210.

13. Bishara SE, Soliman MM, Oonsombat C, Laffoon JF, Ajlouni R (2004) The effect of variation in mesh-base design on the shear bond strength of orthodontic brackets. Angle Orthod 74: 400-404.

14. Klocke A, Kahl-Nieke B (2006) Effect of debonding force direction on orthodontic shear bond strength. Am J Orthod Dentofacial Orthop 129: 261-265.

15. Krell KV, Courey JM, Bishara SE (1993) Orthodontic bracket removal using conventional and ultrasonic debonding techniques, enamel loss, and time requirements. Am J Orthod Dentofacial Orthop 103: 258-266. 
Citation: Yu CC, Yu JH, Lin HJ (2016) An Investigation of the Effects of Chemical Reagents on the Shear Bonding Forces of Orthodontic Metal Brackets. Dentistry 6: 380. doi:10.4172/2161-1122.1000380

Page 6 of 6

16. Boyer DB, Engelhardt G, Bishara SE (1995) Debonding orthodontic ceramic brackets by ultrasonic instrumentation. Am J Orthod Dentofacial Orthop 108: 262-266.

17. Dovgan JS, Walton RE, Bishara SE (1995) Electrothermal debracketing: Patient acceptance and effects on the dental pulp. Am J Orthod Dentofacial Orthop 108: 249-255.

18. Sheridan JJ, Brawley G, Hastings J (1986) Electrothermal debracketing Part I. An in vitro study. Am J Orthod 89: 21-27.

19. Cummings M, Biagioni P, Lamey PJ, Burden DJ (1999) Thermal image analysis of electrothermal debonding of ceramic brackets: in vitro study. Eur J Orthod 21: 111-118.

20. Strobl K, Bahns TL, Williams L, Bishara SE, Stwalley WC (1992) Laseraided debonding of orthodontic ceramic brackets. Am J Orthod Dentofacial Orthop 101: 152-158.

21. Mimura H, Deguchi T, Obata A, Yamagishi T, Ito M (1995) Comparison of different bonding materials for laser debonding. Am J Orthod Dentofacial Orthop 108: 267-273.

22. Mandall NA, Hickman J, Macfarlane TTV, Mattick RCR, Millett DT, et al (2003) Adhesives for fixed orthodontic brackets. The Cochrane Library.

23. Saito K, Sirirungrojying S, Meguro D, Hayakawa T, Kasai K (2005) Bonding durability of using self-etching primer with 4-META/ MMATBB resin cement to bond orthodontic brackets. Angle Orthod 75: 260-265.

24. Nakabayashi N (1992) Adhesive bonding with 4-META. Oper Dent Suppl 5: 125-130.

25. Chang JC, Hurst TL, Hart DA, Estey AW (2002) 4-META use in dentistry: a literature review. J Prosthet Dent 87: 216-224.

26. Clark SA, Gordon PH, McCabe JF (2003) An ex vivo investigation to compare orthodontic bonding using a 4-META-based adhesive or a composite adhesive to acid-etched and sandblasted enamel. Journal of Orthodontics 30: 51-58.

27. Zhiyuan X, Yinglin L (2009) The reactivities of nanoscale colloidal silica toward epoxy compounds and their applications. Christian University, Master Thesis.
28. Wu W, McKinney JE (1982) Influence of chemicals on wear of dental composites. J Dent Res 61: 1180-1183.

29. Larmour CJ, Chadwick RG (1995) Effects of a commercial orthodontic debonding agent upon the surface microhardness of two orthodontic bonding resins. J Dent 23: 37-40.

30. Larmour CJ, McCabe JF, Gordon PH (1998) An ex vivo investigation into the effects of chemical solvents on the debond behaviour of ceramic orthodontic brackets. Br J Orthod 25: 35-39.

31. Erdemir A, Eldeniz AU, Belli S, Pashley DH (2004) Effect of solvents on bonding to root canal dentin. J Endod 30: 589-592.

32. Ezzie E, Fleury A, Solomon E, Spears R, He J (2006) Efficacy of Retreatment Techniques for a Resin-Based Root Canal Obturation Material. J Endod 32: 341-344.

33. Evans DB (1995) Halothane and rouge: An alternative to chloroform and rouge as a disclosing medium. Journal of Prosthetic Dentistry 74: 209-211.

34. Yap AU, Tan SH, Wee SS, Lee CW, Lim EL, et al. (2001) Chemical degradation of composite restoratives. J Oral Rehabil 28: 1015-1021.

35. Arici S, Minors C (2000) The Force Levels Required to Mechanically Debond Ceramic Brackets: An In vitro Comparative Study. Eur J Orthod 22: 327-334.

36. Akhoundi MSA, Mojtahedzadeh F (2005) Problems in Standardization of Orthodontic Shear Bonding forces Tests; A Brief Review. Journal of Dentistry 2: 36-40.

37. Fox NA, McCabe JF, Buckley JG (1994) A critique of bond strength testing in orthodontics. Br J Orthod 21: 33-43.

38. Klocke A, Kahl-Nieke B (2005) Influence of cross-head speed in orthodontic bonding forces testing. Dental Materials 21: 139-144.

39. Chamda RA, Stein E (1996) Time-related bonding forces of light-cured and chemically cured bonding systems: An in vitro study. Am J Orthod Dentofacial Orthop 110: 378-382.

40. Williams VD, Svare CW (1985) The effect of five-year storage prior to bonding on enamel/composite bond strength. J Dent Res 64: 151-154. 